\title{
Leg stiffness and electromyography of knee extensors/flexors: Comparison between older and younger adults during stair descent
}

\author{
Miao-Ju Hsu, PhD; ${ }^{1}$ Shun-Hwa Wei, PhD; ${ }^{2}$ Young-Hue Yu, MD; ${ }^{3}$ Ya-Ju Chang, PhD $^{4 *}$ \\ ${ }^{1}$ Faculty of Physical Therapy, College of Health Science, Kaohsiung Medical University and Department of Rehabilita- \\ tion, Kaohsiung Medical University Hospital, Kaohsiung City, Taiwan; ${ }^{2}$ Graduate Institute of Rehabilitation Science and \\ Technology, National Yang Ming University, Taipei City, Taiwan; ${ }^{3}$ Department of Physical Medicine and Rehabilitation, \\ Taipei City Hospital, Yang Ming Branch, Taipei City, Taiwan; ${ }^{4}$ Physical Therapy Department and Graduate Institute of \\ Rehabilitation Science, Chang Gung University, Taiwan
}

\begin{abstract}
This study investigated differences in knee extensor/flexor electromyography (EMG), leg loading rate, and leg stiffness in older and younger men during stair descent. Sixteen older men (mean $+/-$ standard deviation [SD] $=72+/-$ 4.5 years) and sixteen younger men (mean $+/-\mathrm{SD}=21.2+/-$ 0.5 years) were recruited. The EMG signals were recorded from the rectus femoris and the biceps femoris, while an electrogoniometer measured knee joint angle changes, and a force platform recorded the ground reaction forces (GRFs). We calculated leg stiffness by dividing the first peak GRF by the corresponding leg displacement. We used the Student's $t$-test to examine group differences. Results showed that the older subjects had 78.6\% higher preactivated EMG values in the knee extensor and $128 \%$ greater coactivity in the knee extensors/ flexors in the prelanding phase. At the impact phase, we observed leg stiffness to be $26.6 \%$ greater in the older than the younger subjects. The older subjects needed $94.6 \%$ more support time in performing push-off and also developed a compensatory strategy in stair descent, which altered the scaling of muscle preactivity and increased leg stiffness.
\end{abstract}

Key words: coactivation, elderly, EMG, knee extensor/flexor, leg loading rate, leg stiffness, preactivation, rehabilitation, stair descent, stair walking.

\section{INTRODUCTION}

One of the major problems associated with aging is increased susceptibility to falls. Falls are the leading cause of injury-related hospitalization and death in persons older than 65 [1-3]. Injuries resulting from falls include soft tissue damage and limb fractures [4-5]. Other consequences of falls include decreased mobility, reduced confidence, and death [4].

The largest proportion of falls in elderly people was reported to occur on stairs [6]. The risk of being severely injured may be increased during stair falls because the kinetic energies produced in stair falls may be higher than those from level falls [5]. Svantröm indicated that accidents during stair descent outnumbered those during ascent by more than 3:1 [7]. Nonuniformity of step dimensions [7], inadequate contrast in landing-stair transitions [8], and inadequate visual perception [9] were reported in association with stair falls. Templer et al. further indicated that the majority of stair accidents occurred on the first three and last three steps, irrespective of the length of the stairway [10].

Abbreviations: $\mathrm{BW}=$ body weight, $\mathrm{EMG}=$ electromyography, GRF = ground reaction force, $\mathrm{MVC}=$ maximum voluntary contraction.

*Address all correspondence to Dr. Ya-Ju Chang, Physical Therapy Department and Graduate Institute of Rehabilitation Science; Chang Gung University, 259 Wen-Hwa 1st Road, Kwei-Shan, Tao-Yuan, Taiwan; 886-3-2118800, ext 5515; fax: 886-3-2118800. Email: yichang@mail.cgu.edu.tw DOI: 10.1682/JRRD.2006.04.0033 
Diminished functioning in many physiological systems is believed to contribute to falls in elderly people. Many studies have documented age-related decline in the sensorimotor system that contributes to balance and stability while standing and walking [11-13]. Previous researchers found that successful stair descent required both accurate visualization of the stairs and proper kinesthetic feedback [14-15]. The roles of the somatic and visual systems during stair descent are of special consequence to older adults, as both systems may be impaired in this population [16]. Despite these facts, a smooth stair descent requires good coordination of the agonist and antagonist muscles that regulate joint stiffness during the descent. Since both muscle strength and kinesthetic feedback are diminished by aging, the older individual may use a different control strategy to regulate leg stiffness than the younger individual. Therefore, this study investigated the differences in electromyography (EMG) of knee extensors/flexors, leg loading rate, and leg stiffness in older and younger men during stair descent. We hypothesized that the older mens' gait would have more muscle coactivity and consequently demonstrate a stiffer gait pattern during stair descent.

\section{MATERIALS AND METHODS}

\section{Subjects}

Subjects in this study were 16 younger men, $21.2 \pm 0.5$ years, and 16 older men, $72.0 \pm 4.5$ years old. The younger men were $1.69 \pm 0.05 \mathrm{~m}$ in height and $62.0 \pm 7.5 \mathrm{~kg}$ in body weight, while the older men were $1.61 \pm 0.05 \mathrm{~m}$ in height and $63.5 \pm 9.2 \mathrm{~kg}$ in body weight. Data presented as mean \pm standard deviation. All subjects reported healthy, but sedentary, lifestyles. The younger men were university students and had no known orthopedic or neurological diseases. The older men were required to provide physician's approval before being allowed to participate in this study. Exclusion criteria for the older men included a history of falls and severe orthopedic disease or neurological conditions (i.e., stroke) that prohibited the individuals from descending stairs independently. Test methodology and possible risks were thoroughly explained to each potential subject. Subjects were then requested to read and sign an informed consent document. All experimental procedures met the ethical approval requirements of the National Yang Ming University.

\section{Equipment and Devices}

EMG signals were recorded from the rectus femoris and the biceps femoris of each subject's right leg, with the Motion Lab EMG system (Iomed Inc, Salt Lake City, Utah). The frequency response of the overall system was 40 to $4,000 \mathrm{~Hz}$ as defined by $-3 \mathrm{~dB}$. The EMG electrodes were placed over the muscles of interest following the technique recommended by Zipp [17].

To normalize the EMG signals, we recorded maximum voluntary contraction (MVC) of knee extensors/ flexors while the right knee of the subject was fixed at $45^{\circ}$ of flexion. In this position, the subject was asked to maximally flex or extend his knee against a static resistance for $5 \mathrm{~s}$. To obtain stable maximal force prior to formal EMG data collection, we allowed enough practice time for warm-up and for the subject to be familiar with the testing procedure. We averaged the EMG readings during the middle $3 \mathrm{~s}$ and used them to represent the normalized value (100\% MVC).

We positioned an electrogoniometer (Angle transducer TM5110; TOYO Physical Co, Ltd, Fukuoka, Japan) over the right knee and fastened it to the thigh and shank to monitor the knee joint angle. The potentiometer was calibrated at $0^{\circ}$ and $180^{\circ}$ before use.

The staircase we used in this study is shown in Figure 1. The staircase contained a total of six steps. The force platform, Kistler 9281 (Kistler Instrumente AG, Winterthur, Switzerland), was installed on the concrete floor. We used the height of the force plate plus part of the mounted structure as the first step of the staircase. A wooden man-made stair with five steps was used as the second to sixth steps. Each step in this staircase had a height of $20 \mathrm{~cm}$, a depth of $30 \mathrm{~cm}$, and a width of $130 \mathrm{~cm}$. To protect subjects during stair descent, we placed the

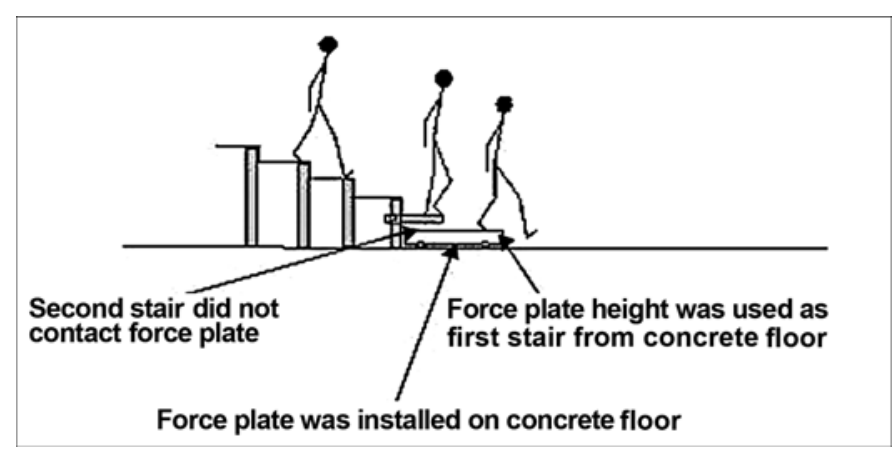

Figure 1.

Schematic of staircase used in stair descent trials. 
staircase next to the wall, and a physical therapist stood on the outer side.

Consecutive EMG, ground reaction force (GRF), and knee joint angle data were simultaneously collected with a data acquisition system (BIOPAC Systems, Inc, Goleta, California) at $1,000 \mathrm{~Hz}$ sampling frequency. All data were analyzed with AcqKnowledge, version 3.5, software (BIOPAC Systems, Inc). We first filtered all EMG signals by a band-pass filter $(20-400 \mathrm{~Hz})$ and then ran them through full-wave rectification. We used the mean value of the middle $3 \mathrm{~s}$ of rectified EMG as the MVC. The rectified EMG data of knee extensors/flexors during stair descent were individually averaged for each phase (prelanding, impact, and push-off) and then normalized to MVC. We calculated the EMG coactivity of the knee extensors/flexors for each phase as the ratio of the mean normalized knee flexors' EMG to the mean normalized knee extensors' EMG. The GRF was normalized to body weight. All force units were transformed to Newtons.

\section{Experimental Protocol}

Each subject dressed in comfortable shorts and wore his personal shoes. Height and weight were measured. The electrogoniometer was placed on the right side of the subject's knee. The rotation center of the electrogoniometer was aligned with the lateral epicondyle.

Each subject participated in one test session of stair descent, in which five trials were recorded (excluding off-balance trials). A rest period of $30 \mathrm{~s}$ was provided between trials. A trial consisted of the subject's stepping off the stair with the right foot and landing with the ball of the right foot on the force plate, without excessive push-off from the upper step by the trailing leg.

To avoid producing negative impact on the stairs, we asked each subject to position his arms neutrally along the body. Arms were not allowed to swing. For safety, an experienced physical therapist stood alongside each subject during descent, but all subjects were able to execute the task without assistance.

\section{Data Recording and Analysis}

To perform quantifiable analysis of the data in stair descent, we divided the movement into three phases: prelanding, impact, and push-off. The prelanding phase was defined as the $250 \mathrm{~ms}$ immediately before foot contact on the force plate. The impact phase was defined as the time interval from initial foot contact on the force plate to the first relative maximum of the GRF. The push- off phase was defined as the time interval from occurrence of the first relative maximum of the GRF to the clearance of the foot from the force plate (Figure 2).

Loading rate was the rate of change in vertical GRF. In this study, the loading rate was defined by final 10 percent minus initial 10 percent of the first relative maximum

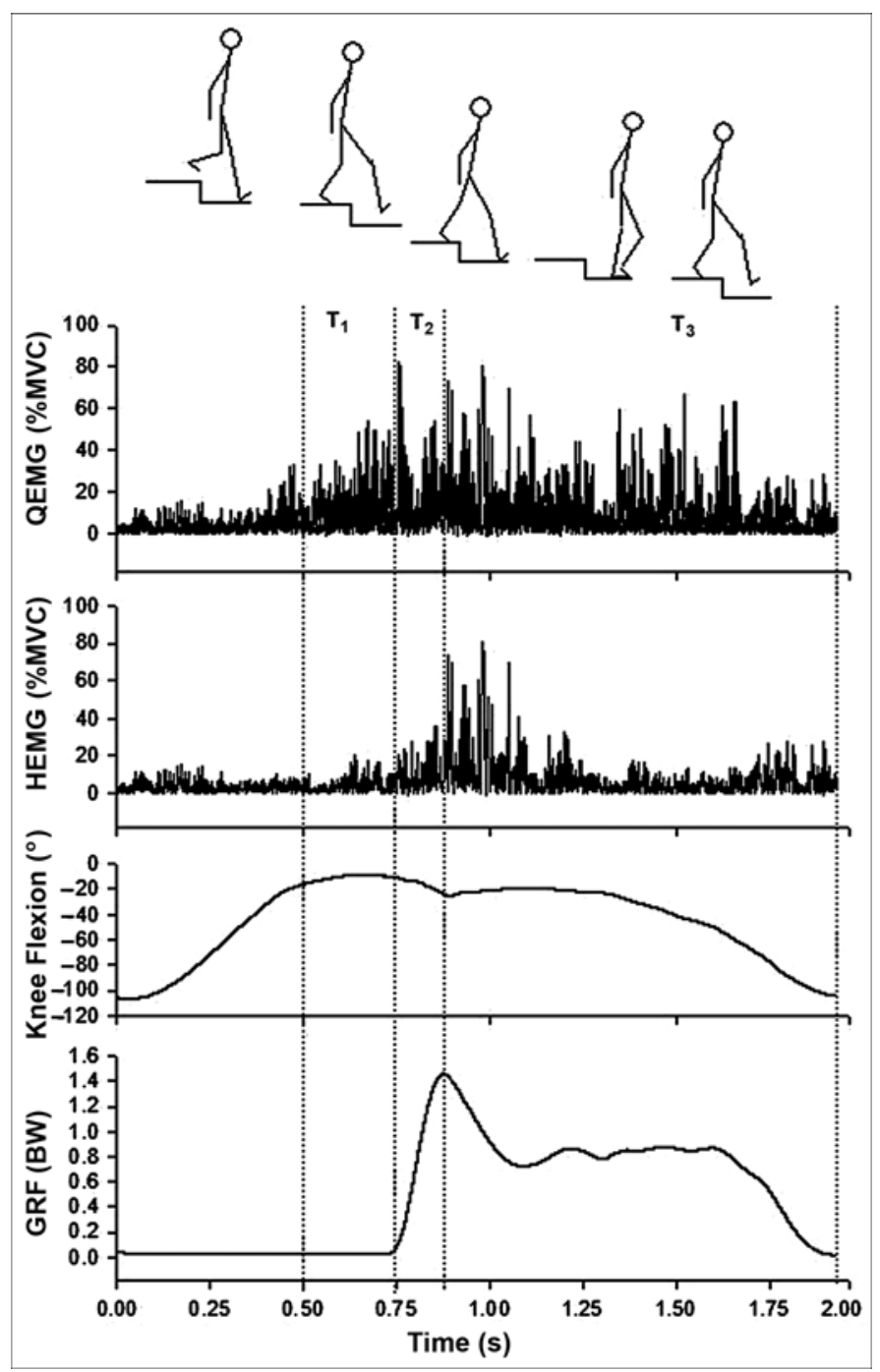

Figure 2.

Stair descent trial for 71-year-old man. Stair descent movement was divided into three phases: prelanding $\left(\mathrm{T}_{1}\right)$, impact $\left(\mathrm{T}_{2}\right)$, and push-off. $\left(T_{3}\right) \cdot T_{1}$ was defined as 250 ms immediately before foot contact on force plate. $T_{2}$ was defined as time interval from initial foot contact on force plate to occurrence of first relative maximum of ground reaction force (GRF). $T_{3}$ was defined as time interval from occurrence of first relative maximum of GRF to clearance of foot from force plate. QEMG and HEMG = electromyography of quadriceps and hamstrings, respectively, $\mathrm{BW}=$ body weight, $\mathrm{MVC}=$ maximum voluntary contraction. 
GRF and then divided by its time interval. Leg length $(L)$ was defined as the distance from the lateral malleolus to the hip joint when the knee was flexed at $0^{\circ}$.

Leg displacement $(\Delta x)$ was computed as the difference between the leg length and the vertical distance from the lateral malleolus to the hip joint. This value was obtained by

$$
\Delta x=L-\left(L_{1}^{2}+L_{2}^{2}-2 L_{1} \times L_{2} \times \cos \theta\right),
$$

where $L_{1}$ was upper-leg length, $L_{2}$ was lower-leg length, and $\theta$ was knee-flexion angle. In this study, leg displacement was expressed as a percentage of each subject's leg length. The lower limb was considered two rigid bodies, thigh and shank. Based on free body diagram concepts [18], the force applied on the hip joint $\left(F_{H}\right)$ was easily obtained by

$$
F_{H}=F_{G}-\left(W_{t}+W_{s}\right) \text {, }
$$

where $F_{G}$ was the value of the vertical GRF and $W_{t}$ and $W_{s}$ were the gravitational force of thigh and shank, respectively. Using peak value of $F_{H}$ at impact phase and its corresponding leg displacement, we obtained the leg stiffness $(k)$ by

$$
k=F_{H} / \Delta x .
$$

In this study, stiffness was normalized by subject's body weight (BW) and leg length. Thus, the unit of leg stiffness was $\mathrm{BW} \cdot \mathrm{L}^{-1}$. The detailed model is shown in Figure 3.

\section{Statistical Analysis}

In this study, the independent variable was age and had two levels, older and younger adults. The dependent variables included peak GRF, loading rate, leg stiffness, and mean rectified EMG of knee extensors/flexors at prelanding, impact, and push-off phases. We used descriptive statistics to understand the features of all measured parameters. We used the Student's $t$-test to examine the differences between the two test groups. The significant difference was set at $\alpha<0.05$. We analyzed the data using the Statistical Package for the Social Sciences software program, version 8.0 (SPSS Inc, Chicago, Illinois).

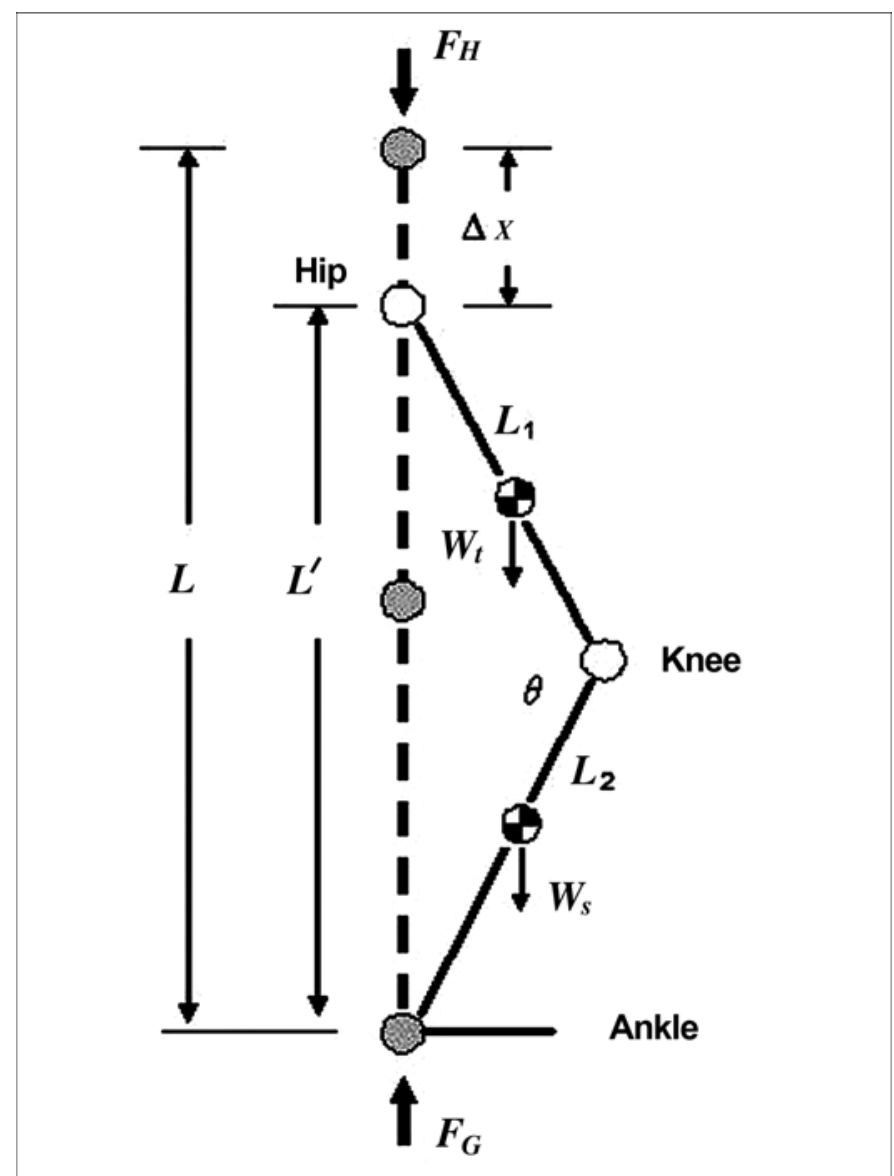

Figure 3.

Biomechanical model for computing leg stiffness during subject stair descent. $\Delta x=$ leg displacement, $L=$ distance from lateral malleolus to hip joint when knee is extended, $L^{\prime}=$ distance from lateral malleolus to hip joint when knee is flexed, $L_{1}=$ upper-leg length, $L_{2}=$ lower-leg length, $\theta=$ knee flexion angle, $F_{G}=$ value of vertical ground reaction force, $F_{H}=$ force applied to hip joint, $W_{t}=$ gravitational force of thigh, $W_{S}=$ gravitational force of shank.

\section{RESULTS}

\section{Prelanding Phase}

Figure 2 shows one stair descent trial for a 71-yearold man. The Table lists maximal GRF, loading rate, leg stiffness, EMG activity, and coactivity data. During the prelanding phase, the older men exhibited 78.6 percent greater preactivity in knee extensors than the younger men. Knee flexor activity observed in the older subjects was also greater than that in younger subjects $(p<0.05)$ (Table). Coactivity of the knee extensors/flexors in the older subjects was also greater than that in the younger subjects by 128 percent. This result indicates that the 
Table.

Maximal ground reaction forces applied to foot, leg stiffness, electromyography (EMG), and muscle coactivity data during stair descent for older $(n=16)$ and younger $(n=16)$ subjects.

\begin{tabular}{|c|c|c|c|c|c|c|}
\hline Parameter & Phase & $\begin{array}{c}\text { Older } \\
\text { Mean } \pm \text { SD }\end{array}$ & $\begin{array}{c}\text { Younger } \\
\text { Mean } \pm \text { SD }\end{array}$ & $\begin{array}{c}\text { Percent } \\
\text { Difference }\end{array}$ & $t$-Test & p-Value \\
\hline Maximal Force (BW) & - & $2.0 \pm 0.4$ & $1.8 \pm 0.3$ & 7.6 & 1.01 & 0.31 \\
\hline Loading Rate $\left(\mathrm{BW} \cdot \mathrm{s}^{-1}\right)$ & - & $29.6 \pm 18.4$ & $15.2 \pm 6.3$ & 94.2 & 2.75 & $0.001^{\dagger}$ \\
\hline Knee Flexion Angle $\left(^{\circ}\right)$ & $\mathrm{T}_{2}$ & $10.6 \pm 14.0$ & $19.5 \pm 8.2$ & 26.6 & -2.67 & $0.01^{\dagger}$ \\
\hline Maximum Leg Displacement $(\% L)$ & $\mathrm{T}_{2}$ & $0.17 \pm 0.01$ & $0.20 \pm 0.01$ & -15.0 & -8.21 & $0.00^{\dagger}$ \\
\hline Maximal Leg Stiffness $\left(\mathrm{BW} \cdot L^{-1}\right)$ & - & $11.7 \pm 3.2$ & $9.2 \pm 6.4$ & 26.5 & 2.38 & $0.02^{\dagger}$ \\
\hline \multirow[t]{3}{*}{ Knee Extensor (\%MVC $\left.{ }^{\ddagger}\right)$} & $\mathrm{T}_{1}$ & $24.6 \pm 12.0$ & $13.8 \pm 5.3$ & 78.6 & 3.07 & $0.02^{\dagger}$ \\
\hline & $\mathrm{T}_{2}$ & $38.7 \pm 11.5$ & $34.3 \pm 11.7$ & 12.3 & 1.00 & 0.32 \\
\hline & $\mathrm{T}_{3}$ & $29.6 \pm 7.7$ & $28.0 \pm 6.6$ & 5.5 & 0.56 & 0.57 \\
\hline \multirow[t]{3}{*}{ Knee Flexor (\%MVC) } & $\mathrm{T}_{1}$ & $23.3 \pm 11.9$ & $6.1 \pm 2.2$ & 279.1 & -2.05 & 0.05 \\
\hline & $\mathrm{T}_{2}$ & $30.1 \pm 8.8$ & $15.5 \pm 6.2$ & 94.3 & -0.21 & 0.83 \\
\hline & $\mathrm{T}_{3}$ & $24.3 \pm 9.1$ & $14.2 \pm 4.8$ & 71.3 & -1.14 & 0.26 \\
\hline \multirow[t]{3}{*}{ Muscle Coactivity ${ }^{\S}$} & $\mathrm{T}_{1}$ & $1.21 \pm 0.71$ & $0.53 \pm 0.26$ & 128.0 & 3.35 & $0.03^{\dagger}$ \\
\hline & $\mathrm{T}_{2}$ & $0.87 \pm 0.42$ & $0.47 \pm 0.21$ & 85.1 & 3.11 & 0.05 \\
\hline & $\mathrm{T}_{3}$ & $0.90 \pm 0.47$ & $0.52 \pm 0.18$ & 73.0 & 2.82 & 0.12 \\
\hline \multirow[t]{2}{*}{ Contact Time (s) } & $\mathrm{T}_{2}$ & $0.32 \pm 0.03$ & $0.35 \pm 0.03$ & -8.6 & -2.25 & $0.03^{\dagger}$ \\
\hline & $\mathrm{T}_{3}$ & $0.72 \pm 0.25$ & $0.37 \pm 0.06$ & 94.6 & 4.99 & $0.001^{\dagger}$ \\
\hline \multicolumn{7}{|c|}{$\begin{array}{l}{ }^{*} \text { Percent difference computed as ((older group - younger group)/younger group) } \times 100 \text {. } \\
{ }^{\dagger} p<0.05 \text {. } \\
{ }^{\ddagger} \text { Maximum voluntary contraction (MVC) expressed as percent of EMG activity measured during maximal isometric quadriceps or hamstring contraction. } \\
\S_{\text {}} \text { Muscle coactivity expressed as ratio of mean normalized knee flexors EMG to mean normalized knee extensor EMG. } \\
\text { BW = body weight, } L=\text { leg length, SD = standard deviation, } \mathrm{T}_{1}=\text { prelanding phase, } \mathrm{T}_{2}=\text { impact phase, } \mathrm{T}_{3}=\text { push-off phase. }\end{array}$} \\
\hline
\end{tabular}

older subjects used greater coactivation of thigh agonist and antagonist muscles during the prelanding phase.

\section{Impact Phase}

Knee flexion angle and leg displacement were less in older subjects compared with younger subjects. This result indicates that older adults performed the stair descent with straighter legs. The older subjects, in comparison with the younger subjects, had only 7.6 percent more maximal force applied to the foot during impact, but this difference was not significant $(p>0.05)$. Leg stiffness was 26.5 percent greater in the older than in the younger subjects $(p<0.05)$. Loading rate of the first peak GRF in older subjects was 94.2 percent greater than for younger subjects. Knee extensor EMG activity at impact showed no significant difference between the older and the younger subjects. Younger subjects had a greater increase in EMG than older subjects during the impact phase compared with the prelanding phase. The older subjects had 8.6 percent less contact time $(p<0.05)$ at the impact phase than the younger subjects. The older subjects also had 85.1 percent greater coactivity in the knee extensors/flexors than the younger subjects.

\section{Push-Off Phase}

The major difference in results between the two test groups during the push-off phase was the amount of contact time. The older subjects required $0.72 \mathrm{~ms}$ of contact time for the push-off phase, while the younger subjects only required $0.37 \mathrm{~ms}(p<0.05)$. The older subjects required 94.6 percent greater contact time for the pushoff phase than the younger subjects. EMG and muscle coactivity showed no significant differences between the two groups during this phase.

\section{DISCUSSION}

Older adults were found to use substantially greater muscle pre- and coactivity during stair descent. Although 
both the older and younger subjects were found to have similar responses in muscle preactivation, EMG readings were 78.6 percent greater for knee extensor muscle control during the prelanding phase for older subjects. Additionally, older subjects were also found to use 128 percent greater muscle cocontraction during the prelanding phase. These data suggest that the older subjects needed greater anticipatory control of the lower-limb muscles when they performed stair descent. These anticipatory activities by the older subjects resulted in preactivation of knee extensor/flexor muscles before foot contact on the stair. Although preactivation in knee extensor/flexor muscles might cause older subjects to have an uncoordinated gait, this anticipatory strategy provided them a safer means of descending stairs. A greater cocontraction of the thigh muscles in older people during stair descent could allow them to generate knee extensor force, increase knee joint stability, and avoid knee buckling at impact phase [1920]. The present study confirmed previous observations that the magnitude of antagonist coactivation increased with age [21].

In this work, we also observed that the older subjects had about 26.5 percent greater leg stiffness during stair descent compared with younger subjects. This result agrees with previous investigations [22-23]. The greater leg stiffness seen in the older adults could result from their contacting the ground with straighter legs, i.e., flexing their knees less (especially at the impact phase), which indicates that they made a conscious or subconscious effort to set the limb position prior to impact. This difference in movement strategy could be interpreted as compensation for significantly reduced muscle strength $[20,24-25]$, a decline in proprioceptive capability $[15,26]$, or a slowed rate of tension development [27]. To reduce the chance of falling, the older adults tended to increase their leg stiffness by cocontracting knee extensors/flexors and decreasing their knee flexion angles. This helped them anticipate a safer landing, but this posture results in greater reliance on the skeletal system and less reliance on the muscles for impact absorption during landing [28-29]. The older adults needed greater thigh muscle cocontraction and straighter legs for landing in stair descent, which caused them to produce a higher loading rate on the lower limb. Rapid knee flexion at impact phase would decrease peak GRF because rapid knee flexion reduces effective mass of the lower limbs. On the contrary, maintaining a straighter leg at impact phase might increase peak GRF because effective mass of the lower limb remains constant. In our study, we found that older adults maintained less knee flexion angle and straighter legs at the impact phase of stair descent. These actions might result in a higher loading rate on their lower limbs.

At push-off phase, the subjects were preparing the leg for the next downward step. To decrease sudden GRF applied on the lower limb, the older adults' strategy was to increase push-off time on the other leg. This allowed the landing leg to have much more time for subsequent foot-to-floor contact. This compensatory mechanism could be also associated with degradation of muscle strength and a slower rate of muscle force production $[20,25,30]$.

\section{CONCLUSIONS}

Older adults developed different strategies for stair descent than the younger adults. These strategies included increased muscle pre- and coactivity at the prelanding phase, decreased knee flexion angle and leg displacement, increased leg stiffness and loading rate at the impact phase, and increased support time at the pushoff phase. Although this strategy may help the older adults anticipate a safer stair descent, this posture results in greater reliance on the skeletal system and less reliance on muscle system. An exercise program that emphasizes reconditioning muscle strength and increasing the tension development rate may help older individuals improve neuromotor control during stair descent.

\section{ACKNOWLEDGMENTS}

This material is the result of work partially supported with resources from the Ministry of Education, Republic of China (Taiwan), Aim for the Top University Plan.

The authors have declared that no competing interests exist.

\section{REFERENCES}

1. Campbell AJ, Reinken J, Allan BC, Martinez GS. Falls in old age: A study of frequency and related clinical factors. Age Ageing. 1981;10(4):264-70. [PMID: 7337066$]$ 
2. Campbell AJ, Borrie MJ, Spears GF. Risk factors for falls in a community-based prospective study of people 70 years and older. J Gerontol. 1989;44(4):M112-17. [PMID: 2738307]

3. Centers for Disease Control and Prevention. Nonfatal fallrelated traumatic brain injury among older adults - California, 1996-1999. MMWR Morb Mortal Wkly Rep. 2003; 52(13):276-78. [PMID: 12729076]

4. The prevention of falls in later life. A report of the Kellogg International Work Group on the prevention of falls by the elderly. Dan Med Bull. 1987;34 Suppl 4:1-24. [PMID: 3595217]

5. Luukinen H, Herala M, Koski K, Honkanen R, Laippala P, Kivela SL. Fracture risk associated with a fall according to type of fall among the elderly. Osteoporos Int. 2000;11(7): 631-34. [PMID: 11069199]

6. Nie NH, Hull CH, Jenkins JG, Steinbrenner KS, Ben DH. National Safety Council: Accident facts. New York (NY): McGraw-Hill Books; 1983.

7. Svantröm L. Falls on stairs: An epidemiological accident study. Scand J Soc Med. 1974;2(3):113-20. [PMID: 4432054$]$

8. Carstens DY. Site planning and design for the elderly: Issues, guidelines, and alternatives. New York (NY): Van Nostrand Reinhold; 1985. p. 170.

9. Green I. Housing for the elderly: The development and design process. New York (NY): Van Nostrand Reinhold; 1975. p. 174.

10. Templer J, Mullet GM, Archea J. An analysis of the behavior of stair users. Washington (DC): National Technical Information Service; 1978. p. 13.

11. Ducan G, Wilson JA, MacLennan WJ, Lewis S. Clinical correlates of sway in elderly people living at home. Gerontology. 1992;38(3):160-66. [PMID: 1624144]

12. Lord SR, Clark RD, Webster IW. Postural stability and associated physiological factors in a population of aged persons. J Gerontol. 1991;46(3):M69-76. [PMID: 2030269]

13. Lord SR, Ward JA. Age-associated differences in sensorimotor function and balance in community dwelling women. Age Aging. 1994;23(6):452-60. [PMID: 9231937]

14. Patla AE, Rietdyk S. Visual control of limb trajectory over obstacles during locomotion: Effect of obstacle height and width. Gait Posture. 1993;1:45-60.

15. Woollacott MH, Tang PF. Balance control during walking in the older adult: Research and its implications. Phys Ther. 1997;77(6):646-60. [PMID: 9184689]

16. Inglin B, Woollacott MH. Age-related changes in anticipatory postural adjustments associated with arm movements. J Gerontol. 1988;43(4):M105-13. [PMID: 3385142]

17. Zipp P. Recommendations for the standardization of lead position in surface electromyography. Eur J Appl Physiol. 1982;50:41-54.

18. Winter DA. Biomechanics and motor control of human movement. 2nd ed. New York (NY): John Wiley \& Sons; 1990.
19. Gollhofer A, Schmidtbleicher D, Dietz V. Regulation of muscle stiffness in human locomotion. Int J Sports Med. 1984;5(1):19-22. [PMID: 6698678]

20. Blanpied P, Smidt GL. The difference in stiffness of the active plantarflexors between young and elderly human females. J Gerontol. 1993;48(2):M58-63. [PMID: 8473695$]$

21. Hakkinen K, Alen M, Kallinen M, Izquierod M, Jokelainen K, Lassila H, Malkia E, Kraemer WJ, Newton RU. Muscle CSA, force production, and activation of leg extensor muscle during isometric and dynamic actions in middle-aged and elderly men and women. J Aging Phys Activ. 1998; 6(3):232-47.

22. Hortobagyi T, DeVita P. Altered movement strategy increases lower extremity stiffness during stepping down in the aged. J Gerontol A Biol Sci Med Sci. 1999;54(2):B63-70. [PMID: 10051852]

23. Hortobagyi T, DeVita P. Muscle pre- and coactivity during downward stepping are associated with leg stiffness in aging. J Electromyogr Kinesiol. 2000;10(2):117-26. [PMID: 10699559]

24. Brown M, Fisher JS, Salsich G. Stiffness and muscle function with age and reduced muscle use. J Orthop Res. 1999; 17(3):409-14. [PMID: 10376731]

25. Grabiner MD, Enoka RM. Changes in movement capabilities with aging. Exerc Sport Sci Rev. 1995;23:65-104.

[PMID: 7556361]

26. Skinner HB, Barrack RL, Cook SD. Age-related declines in proprioception. Clin Orthop Relat Res. 1984;(184):208-11. [PMID: 6705349]

27. Man'kovskii NB, Mints AY, Lysenyuk VP. Regulation of the preparatory period for complex voluntary movement in old and extremely old age. Hum Physiol. 1980:6(1):46-50. [PMID: 7399536]

28. DeVita P, Hortobagyi T, Barrier J, Money J, Anderson E. Effects of aging on skeletal and muscular components of lower extremity quasi-stiffness. In: Proceedings of the 21st Annual Meeting of the American Society of Biomechanics; 1997 Sep 24-27; Clemson, SC. American Society of Biomechanics; 1997. p. 282-83.

29. Burke JR, Kamen G. Impairments of the response preparation process in elderly. Int J Neurosci. 1995;81(3-4):177-92. [PMID: 7628909]

30. Burke JR, Kamen G. Changes in spinal reflexes preceding a voluntary movement in young and old adults. J Gerontol A Biol Sci Med Sci. 1996;51(1):M17-22. [PMID: 8548508]

Submitted for publication April 7, 2006. Accepted in revised form January 23, 2007. 